Received: 23 February 2018

Accepted: 16 July 2018

Published online: 22 August 2018

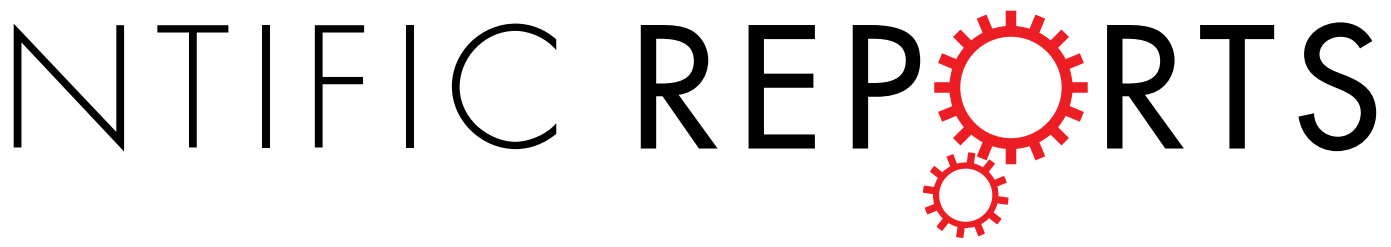

\title{
OPEN Targeting protein-protein interactions for therapeutic discovery via FRET-based high- throughput screening in living cells
}

Daniel R. Stroik ${ }^{1}$, Samantha L. Yuen ${ }^{1}$, Kevyn A. Janicek ${ }^{1}$, Tory M. Schaaf ${ }^{1}$, Ji Li ${ }^{1}$, Delaine K. Ceholski ${ }^{2}$, Roger J. Hajjar ${ }^{2}$, Razvan L. Cornea $\mathbb{D}^{1}$ \& David D. Thomas ${ }^{1}$

We have developed a structure-based high-throughput screening (HTS) method, using time-resolved fluorescence resonance energy transfer (TR-FRET) that is sensitive to protein-protein interactions in living cells. The membrane protein complex between the cardiac sarcoplasmic reticulum Ca-ATPase (SERCA2a) and phospholamban (PLB), its Ca-dependent regulator, is a validated therapeutic target for reversing cardiac contractile dysfunction caused by aberrant calcium handling. However, efforts to develop compounds with SERCA2a-PLB specificity have yet to yield an effective drug. We coexpressed GFP-SERCA2a (donor) in the endoplasmic reticulum membrane of HEK293 cells with RFPPLB (acceptor), and measured FRET using a fluorescence lifetime microplate reader. We screened a small-molecule library and identified 21 compounds (Hits) that changed FRET by $>3$ SD. 10 of these Hits reproducibly alter SERCA2a-PLB structure and function. One compound increases SERCA2a calcium affinity in cardiac membranes but not in skeletal, suggesting that the compound is acting specifically on the SERCA2a-PLB complex, as needed for a drug to mitigate deficient calcium transport in heart failure. The excellent assay quality and correlation between structural and functional assays validate this method for large-scale HTS campaigns. This approach offers a powerful pathway to drug discovery for a wide range of protein-protein interaction targets that were previously considered "undruggable".

A major goal of drug discovery in recent years is the development of small molecules that target specific protein-protein interactions ${ }^{1}$, but there is a growing consensus that such targets are intrinsically difficult to perturb specifically with small molecules ${ }^{2}$. In the present study, we focus on the interaction of phospholamban (PLB), a 52-residue single-pass transmembrane protein expressed in the sarcoplasmic reticulum (SR) of cardiac muscle, and its regulatory target SERCA2a, the cardiac SR Ca-ATPase. SERCA2a is responsible for removing Ca from the cytosol into the SR, inducing muscle relaxation ${ }^{3}$. In its enzymatic cycle, the Ca-ATPase undergoes a transition from a high Ca affinity (E1) to a low Ca affinity (E2) conformation, with ATP binding and autophosphorylation powering the calcium transport process. PLB is in dynamic equilibrium between homopentamers and monomers, where the oligomeric state is proposed to act as a reservoir ${ }^{4}$. Monomeric PLB reduces the apparent Ca affinity of SERCA2a, and this inhibitory effect is relieved by $\beta$-adrenergic stimulation of PLB phosphorylation, thus providing a Ca-transport reserve to enhance cardiac performance. In heart failure (HF), however, there is a Ca-transport deficit that leads to elevated sarcoplasmic $\mathrm{Ca}$ (cytotoxic) and incomplete relaxation and filling of the ventricle (diastolic dysfunction), as well as incomplete SR re-filling with Ca, which blunts Ca release (systolic dysfunction $)^{5}$. The market is saturated with preload and afterload reducers that provide symptomatic relief, but there is an urgent need for an effective and safe cardiotonic therapy that directly targets deteriorated diastolic and systolic function. Since HF depends on multiple factors and causes, numerous strategies have been developed to mitigate or reverse cardiac dysfunction. A promising approach is to enhance cardiac muscle contractility by modulating Ca transport ${ }^{6,7}$. The SERCA2a-PLB interaction is widely viewed as an attractive target for cardiovascular therapeutic discovery and development, to correct the pathophysiological myocyte state and its consequences to cardiac function.

${ }^{1}$ Department of Biochemistry, Molecular Biology, and Biophysics, University of Minnesota, Minneapolis, Minnesota, 55455 , USA. ${ }^{2}$ Cardiovascular Research Center, Icahn School of Medicine at Mount Sinai, New York City, New York, 10029, USA. Correspondence and requests for materials should be addressed to D.D.T. (email: ddt@umn.edu) 
a

\section{SERCA2a-PLB Biosensor}

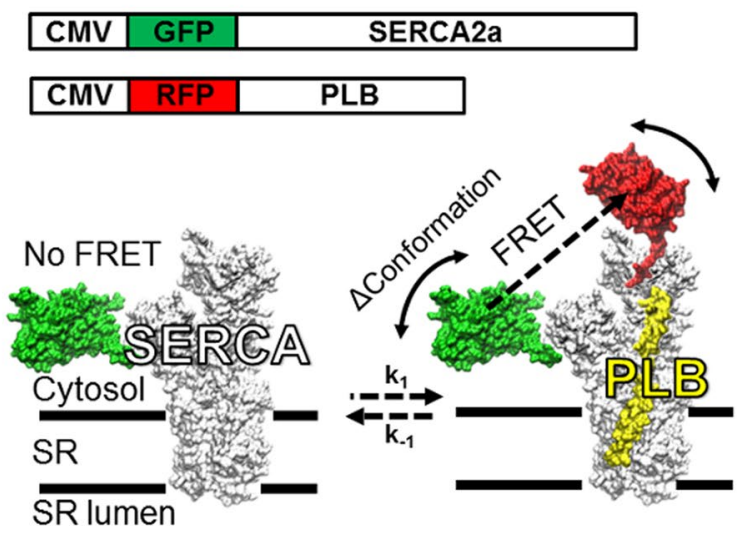

b

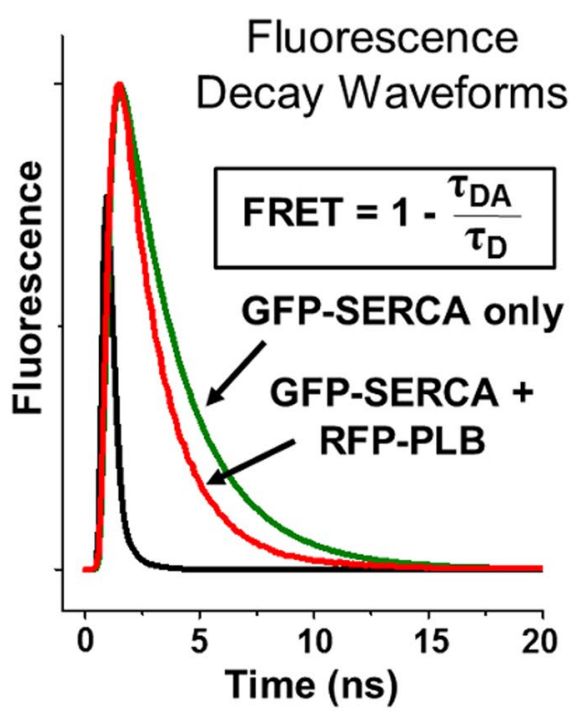

Figure 1. Structure-based high-throughput screening to target SERCA2a-PLB complex. (a) Schematic diagram illustrating expression vectors and structural model of GFP-SERCA2a free or bound to RFP-PLB. (b) Timeresolved fluorescence waveforms as a readout to measure FRET between GFP-SERCA2a and RFP-PLB. FRET is calculated as the change between lifetimes (exponential decay times) of donor-only and donor-acceptor samples $\left(\tau_{\mathrm{D}}\right.$ and $\left.\tau_{\mathrm{DA}}\right)$.

It is well established that decreased SERCA2a activity, as seen in HF animal models and human patients, results in slower and less complete muscle relaxation after each contraction ${ }^{8-11}$. Recent efforts using gene therapy to increase SERCA2a activity accomplish this either through SERCA2a overexpression or by reducing SERCA2a inhibition by $\mathrm{PLB}^{12,13}$. SERCA2a activation is tolerated in healthy animal models and significantly enhances cardiac function in numerous models of heart disease ${ }^{14,15}$. These results validate SERCA2a activation for HF therapy. SERCA2a overexpression via recombinant adeno-associated virus (rAAV) was achieved in patients experiencing end-stage HF in a recent phase II clinical trial ${ }^{16}$. Despite encouraging preliminary results ${ }^{17}$, the trial failed to meet its primary end goals, due to dosage constraints and fundamental limitations of rAAV gene therapy; e.g., in patients with pre-existence of neutralizing antibodies ${ }^{18}$.

We have pursued an alternative approach to activate SERCA2a using small-molecule drugs that decrease SERCA2a inhibition by PLB. This small-molecule approach is designed to overcome limitations associated with gene therapy ${ }^{18}$ and is amenable to acute, non-invasive hospital intervention with the potential for chronic usage to improve cardiac contractility. As PLB is almost exclusively expressed in the heart ${ }^{4}$, compounds that specifically target the SERCA2a-PLB complex will be inherently tissue-specific, thus reducing the risk of adverse side effects. Some previous attempts to discover compounds that activate SERCA have been largely unsuccessful, due to reliance on low-throughput, low-precision assays of ATPase activity ${ }^{19}$. We previously reported a fluorescence resonance energy transfer (FRET)-based high-throughput screening (HTS) method using purified SERCA and PLB labeled with fluorescent dyes in a reconstituted membrane system ${ }^{20}$. A 20,000-compound screen was performed on this in vitro sample using steady-state (intensity) fluorescence detection and identified the first SERCA activators. Surprisingly, none of these compounds directly affected the SERCA2a-PLB interaction ${ }^{20}$. We hypothesize that detection of compounds that disrupt the SERCA2a-PLB requires a more precise detection technique.

Here, we introduce an HTS platform that directly monitors the SERCA2a-PLB interaction using time-resolved FRET (TR-FRET) between GFP-tagged SERCA2a and RFP-tagged PLB constructs expressed in a transformed human cell line (Fig. 1a). When RFP-PLB (acceptor) is bound to GFP-SERCA2a (donor), FRET is detected as a decrease in donor fluorescence lifetime (FLT), which is calculated from fluorescence decay waveforms (Fig. 1b). The FRET measurement is a direct readout of changes in PLB binding to SERCA2a and/or the structure of the SERCA2a-PLB complex. The $\mathrm{R}^{-6}$ distance dependence of FRET makes it sensitive to protein-protein interactions and subtle structural changes. We applied this structure-based SERCA2a-PLB biosensor to drug discovery, by performing a triplicate screen of the Library of Pharmacologically Active Compounds (LOPAC, 1280 compounds), using an FLT plate reader provided by Fluorescence Innovations, Inc ${ }^{21,22}$. The FLT measurement yields a 30 -fold decrease in the coefficient of variation $(\mathrm{CV}=$ standard deviation/mean) compared with intensity detection used in our previous screen ${ }^{20}$, resulting in an HTS platform of excellent quality. To assess the effectiveness of this HTS platform, we performed FRET concentration-response and ATPase activity assays, revealing ten Hit compounds that reproducibly affect SERCA2a-PLB structure and function. This strong correlation between structural and functional assays validates this method to discover SERCA2a-PLB effectors for large-scale HTS campaigns. 

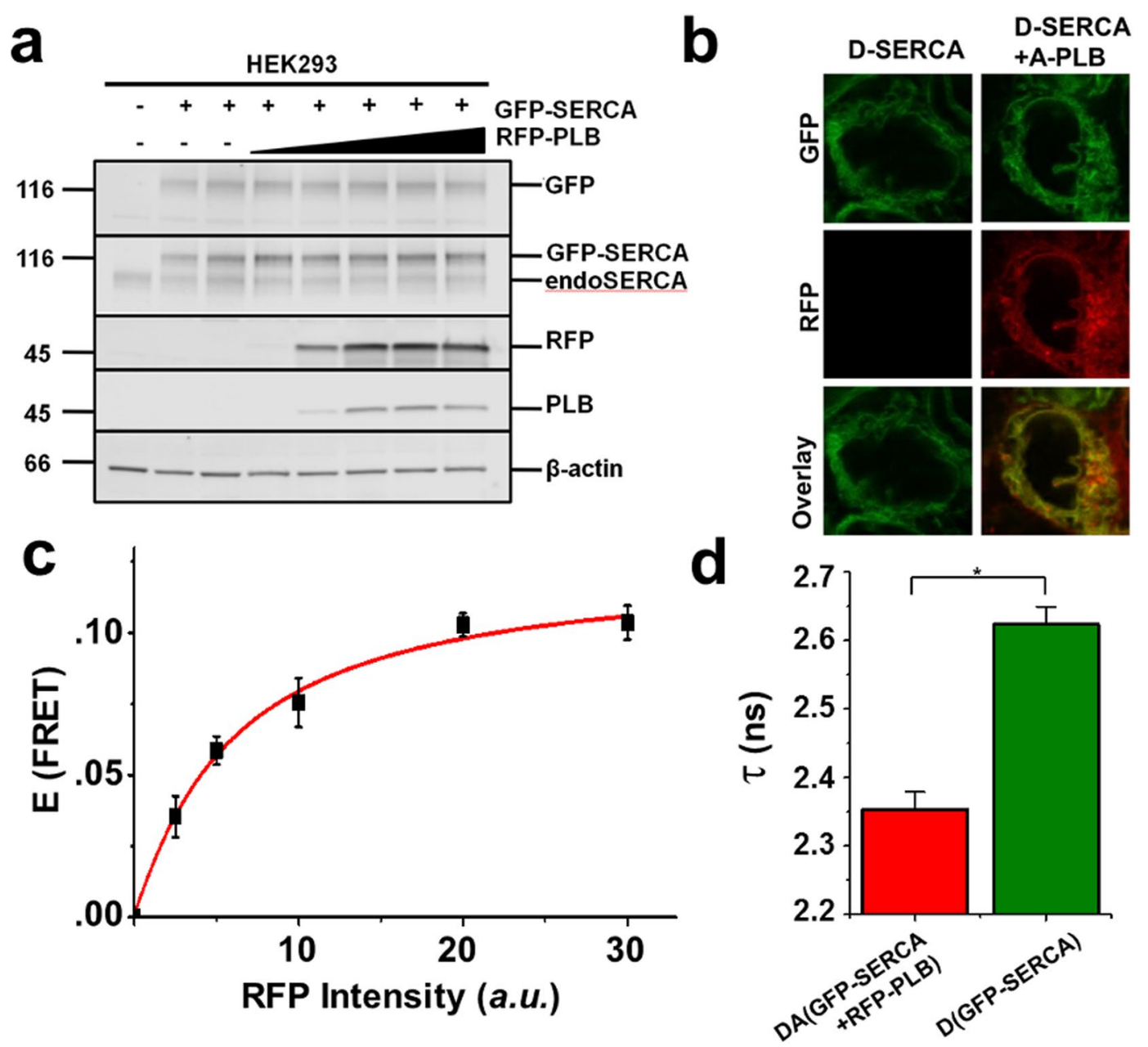

Figure 2. Biochemical and spectroscopic characterization of SERCA2a-PLB biosensor. (a) Immunoblots of homogenates from untransfected HEK293 cells (lane 1), cells expressing GFP-SERCA2a (lanes 2-3), or cells expressing GFP-SERCA2a and increasing amounts of RFP-PLB (lanes 4-8). Antibodies from top to bottom are anti-GFP, anti-SERCA2, anti-RFP, anti-PLB and anti- $\beta$-actin, and the image has been cropped. (b) Confocal fluorescence imaging of HEK293 cells expressing GFP-SERCA2a (left) or GFP-SERCA2a and RFP-PLB (right). (c) FRET efficiency E from GFP-SERCA2a (donor) to RFP-PLB (acceptor) shows hyperbolic dependence on acceptor concentration. (d) The mean FLT in the presence of saturating acceptor is $\tau_{\mathrm{DA}}=2.35 \pm 0.02 \mathrm{~ns}$, corresponding to $\mathrm{E}=0.103 \pm 0.004$. Error bars indicate $\mathrm{SD}(\mathrm{n}=3)$. Statistical differences determined using the Student's t test: $* \mathrm{P} \leq 0.01$.

\footnotetext{
Results

Expression, localization, and FRET readout of SERCA2a-PLB biosensor. To monitor the SERCA2a-PLB interaction in a natural membrane environment, we developed a biosensor system by fusing GFP and RFP to the N-termini of SERCA2a and PLB, respectively, and measured FRET changes as a readout of changes in the SERCA2a-PLB complex structure and binding. Attaching fluorescent proteins (FP) at these sites does not interfere with the activity of SERCA or PLB ${ }^{23}$. HEK293 cells were transfected with GFP-SERCA2a and RFP-PLB constructs, and expression was verified by SDS-PAGE and immunoblot (Fig. 2a). The GFP-tagged SERCA protein was resolved from untagged SERCA, as verified by a GFP-specific antibody. There were no other bands of lower mobility, and there was no aggregation apparent in intact cells. To specifically detect the SERCA2a-PLB interaction, a monomeric mutant of $\mathrm{PLB}^{24}$ was used, with three mutations $(\mathrm{C} 36 \mathrm{~A}, \mathrm{C} 41 \mathrm{~F}$, and $\mathrm{C} 46 \mathrm{~A})$ in the transmembrane domain. The structure and function of this PLB variant is indistinguishable from those of monomeric wild-type PLB ${ }^{25,26}$. Transfection of this RFP-PLB variant produced a single band recognized by PLB- and RFP-specific antibodies. The SERCA2a-PLB biosensor showed the expected endoplasmic reticulum localization by confocal microscopy (Fig. 2b) with no bright puncta (which would have indicated aggregation) or other non-uniformities. FRET between GFP-SERCA2a and RFP-PLB in live cells showed hyperbolic dependence on protein concentration, with a maximum energy transfer efficiency E (fractional decrease of FLT or intensity) of $0.103 \pm 0.004$ (Fig. 2c,d). The observed nanosecond time-resolved fluorescence decay was best fitted by a two-Gaussian distance distribution, centered at $R_{1}=5.6 \pm 1.6 \mathrm{~nm}$ and $R_{2}=9.8 \pm 1.9 \mathrm{~nm}$ (Figure $\left.\mathrm{S} 1\right)^{27-29}$. The measured distances are in agreement with previous TR-FRET measurements ${ }^{30}$.
} 

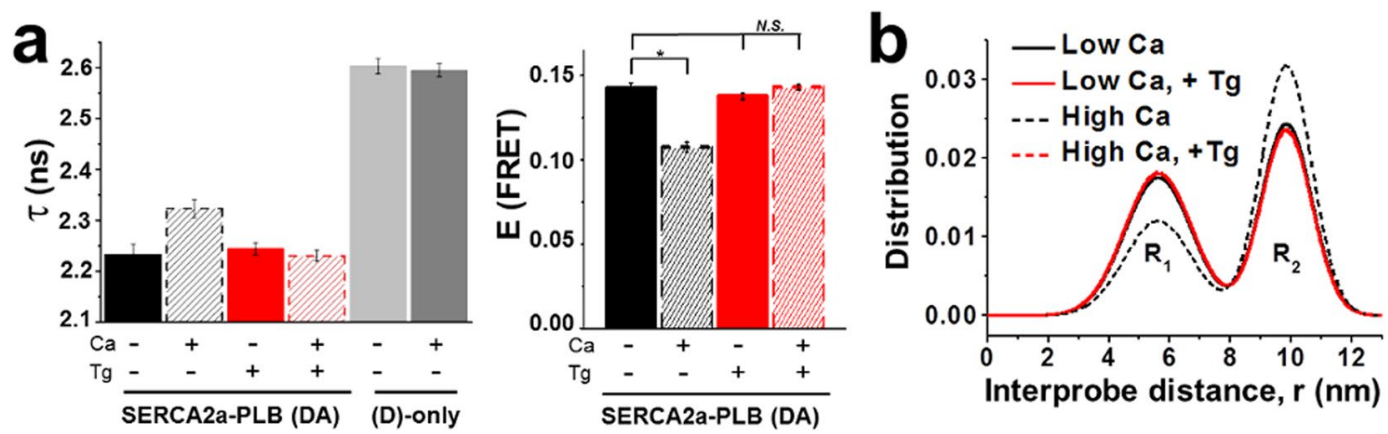

Figure 3. Effects of $\mathrm{Ca}$ and Tg on SERCA2a-PLB biosensor. (a) Left- FLT measurements in saponinpermeabilized cells under conditions of low $\mathrm{Ca}(100 \mathrm{nM})$ and high $\mathrm{Ca}(10 \mu \mathrm{M})$, and in the absence and presence $(100 \mathrm{nM})$ of the SERCA inhibitor thapsigargin (Tg). Right- FRET readout calculated from (a) shows a Ca-dependent decrease in FRET, that is ablated by addition of Tg. Error bars indicate SD $(n=3)$. Statistical differences determined using 1-way ANOVA followed by Tukey post hoc analysis (more detailed analysis is provided in Supplemental Table 4). $* \mathrm{P} \leq 0.01$ and N.S. indicating $\mathrm{P} \geq 0.05$. (b) Distance distributions between donor on SERCA and acceptor on PLB, based on Eq. (10).
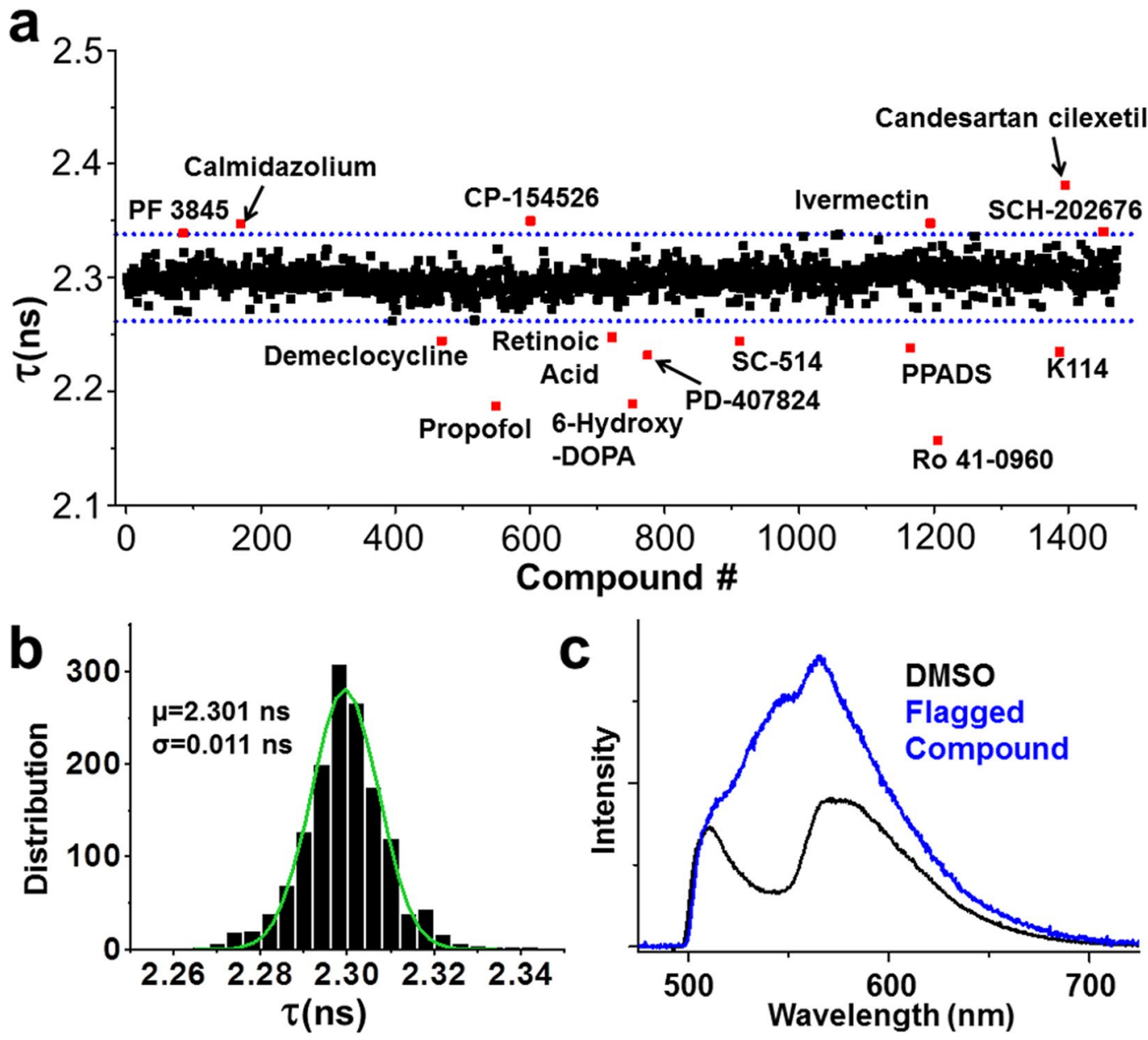

Figure 4. High-throughput screen results. Compounds were screened in triplicate at a final concentration of $10 \mu \mathrm{M}$. (a) FLT values from one representative screen including DMSO control wells with a Hit threshold ( $>$ 3 S.D. of mean) indicated by dotted blue lines. (b) Gaussian fit of the FLT distribution. (c) SERCA2a-PLB biosensor visible emission spectrum upon excitation at $473 \mathrm{~nm}$ (black). Addition of a fluorescent compound greatly alters the spectrum (blue). 
a

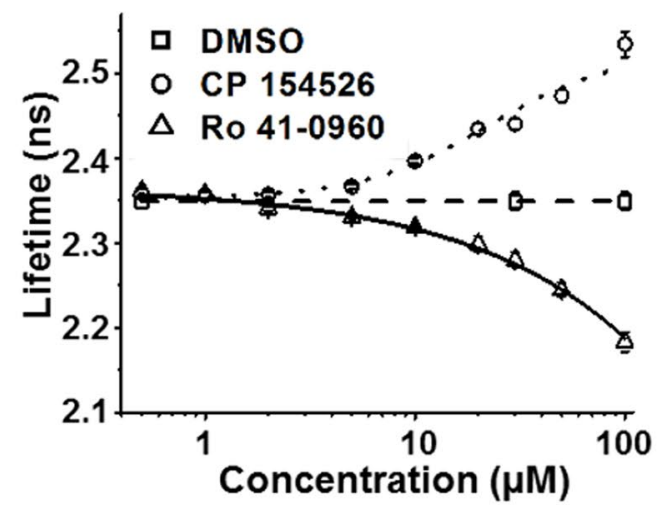

\section{Ro 41-0960}

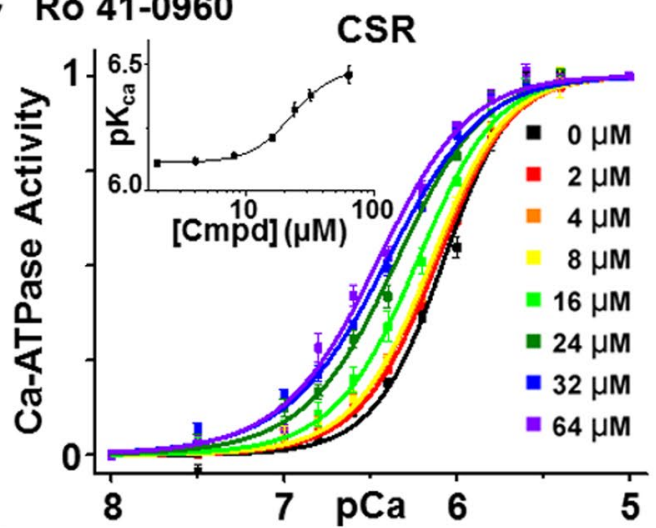

d

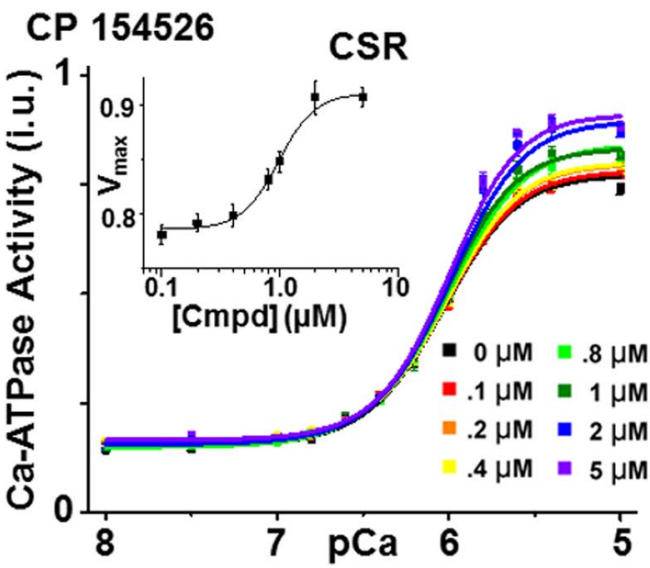

b Ro 41-0960
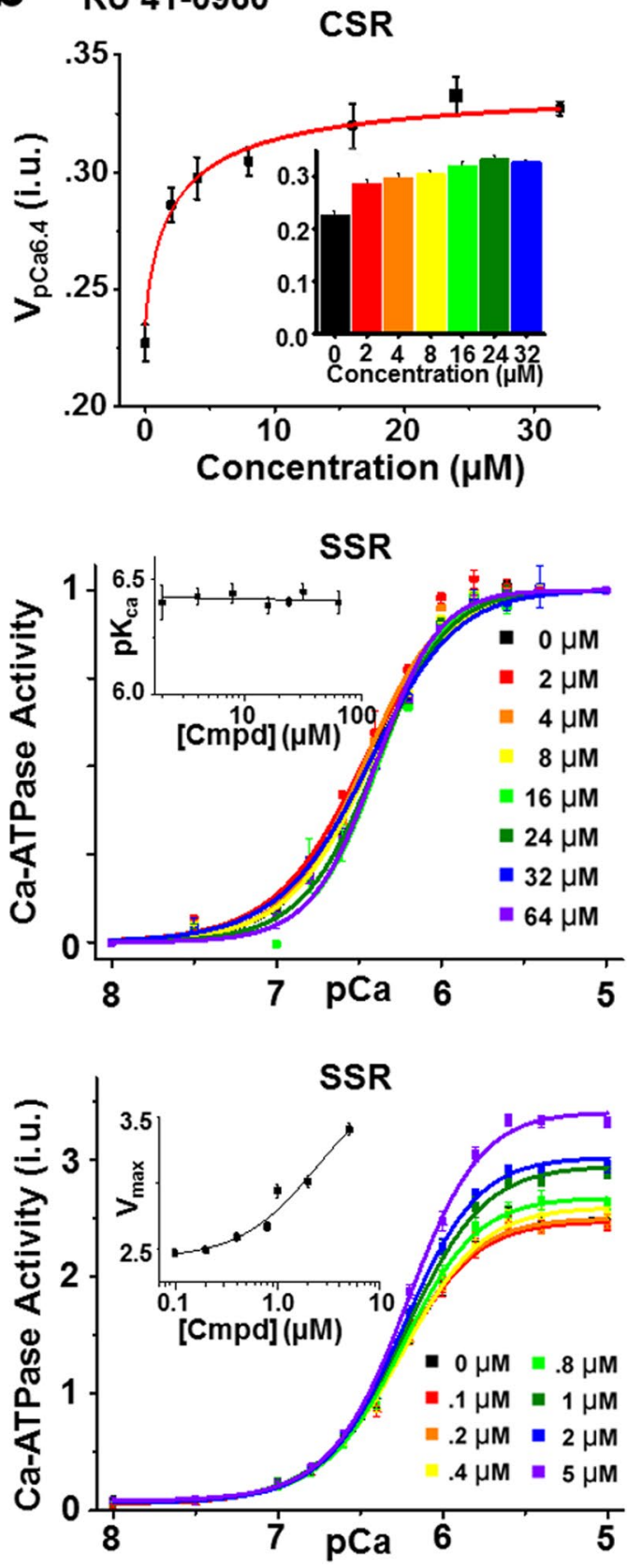

Figure 5. Effects of SERCA activators identified in this screen. (a) FRET dependence on $(0.01-100 \mu \mathrm{M}) \mathrm{CP}$ 154526 (circle) and Ro 41-0960 (up-triangle) under conditions similar to those in the primary HTS. (b) (inset) Dependence of CSR Ca-ATPase activity at $\mathrm{pCa} 6.4$ on the concentration of Ro 41-0960. Error bars indicate $\mathrm{SD}(\mathrm{n}=3)$. Statistical differences determined using 1-way ANOVA followed by Tukey post hoc analysis (more detailed analysis is provided in Supplemental Table 5). (c) Normalized Ca-ATPase activity of cardiac SR (CSR) and skeletal SR (SSR) was measured after a $20 \mathrm{~min}$ incubation in the presence of Ro 41-0960 (up to $64 \mu \mathrm{M}$ ) or DMSO control. Error bars indicate SEM $(n=3)$. (inset) Concentration response of SERCA apparent Ca affinity $\left(\mathrm{pK}_{\mathrm{Ca}}\right.$ ). (d) Ca-ATPase activity of CSR and SSR after $20 \mathrm{~min}$ incubation in the presence of CP 154526 (up to $5 \mu \mathrm{M})$ or DMSO control. Error bars indicate SEM $(n=3)$. (inset) Concentration response for maximum velocity (V at pCa $5 ; \mathrm{V}_{\max }$ ).

PLB inhibition of SERCA is relieved by elevated $\mathrm{Ca}^{4}$. The most likely explanation, supported by in vitro measurements of FRET between SERCA and PLB, is that relief of inhibition occurs primarily through a structural

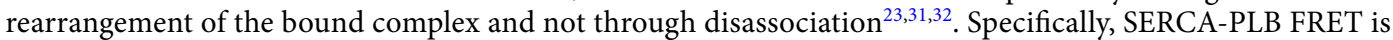
reduced in the presence of a high Ca concentration but is not ablated ${ }^{23,31}$. To test this, we quantified FRET changes in permeabilized cells under conditions of low $\mathrm{Ca}(100 \mathrm{nM})$ and high $\mathrm{Ca}(10 \mu \mathrm{M})$. In agreement with previous studies, high $\mathrm{Ca}$ resulted in a modest FRET decrease (from $\mathrm{E}=0.14$ to $\mathrm{E}=0.11$ ), confirming that the binding 

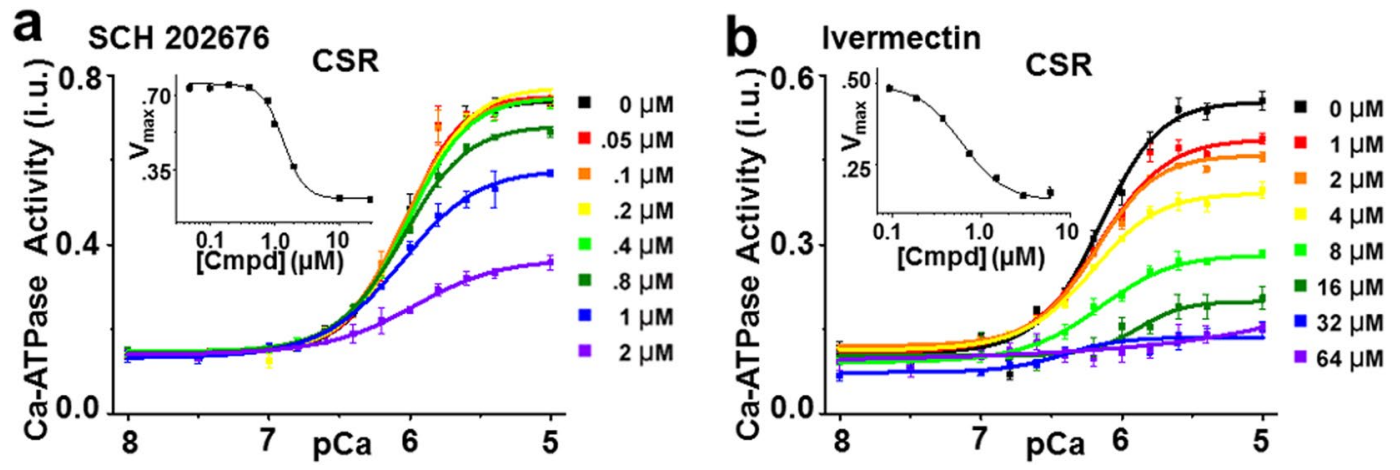

Figure 6. Ca-ATPase activity for SERCA inhibitors. (a) ATPase activity from CSR) was measured after 20 min incubation with SCH 202676 (up to $2 \mu \mathrm{M}$ ) or DMSO control. (inset) Concentration response for limiting activity at high $\mathrm{Ca}\left(\mathrm{V}_{\max }\right)$. (b) ATPase activity from CSR after $20 \mathrm{~min}$ incubation in the presence of ivermectin (up to $64 \mu \mathrm{M}$ ) or DMSO control. (inset) Concentration response for limiting activity at high $\mathrm{Ca}\left(\mathrm{V}_{\max }\right)$.

interface is altered by structural changes but that the SERCA2a-PLB complex remains intact (Fig. 3a). Treatment with thapsigargin (Tg), a SERCA inhibitor that shifts the transition between the E1/E2 conformations toward E2, prevents the Ca-dependent decreases in FRET (Fig. 3a). We consistently observe a decreased DA lifetime in saponin-treated cells and cell homogenates relative to the live cell assay (Fig. 2d); this may reflect changes in membrane dynamics or the fluorophore environment ${ }^{33}$. As both $\mathrm{Tg}$ and low Ca promote the $\mathrm{E} 2$ conformation, we hypothesized that we would be able to resolve the distance distribution associated with this conformational state by TR-FRET. Indeed, we see a shift in the equilibrium of the structural states from the short distance $\left(\mathrm{R}_{1}\right)$ to the long distance $\left(\mathrm{R}_{2}\right)$ under high-Ca conditions, and this is ablated by Tg (Fig. 3b). This demonstrates that the SERCA2a-PLB biosensor is sensitive to changes in the protein complex interactions, and thus suitable for our HTS platform.

HTS of LOPAC library to identify compounds that affect SERCA2a-PLB FRET. To assess the performance of our FRET-based HTS method, we screened a library of 1280 small-molecule compounds (LOPAC) for identification of SERCA2a-PLB modulators. This library is suitable for a pilot screen, as it covers all major drug target classes and most compounds are commercially available, satisfactory for the requirements of orthogonal screening ${ }^{34}$. Assay quality was determined based on controls (DMSO-only samples) on each plate, as indexed by the Z' factor from Eq. $(2)^{21,35}$. The high precision enabled using FLT measurements provides an excellent HTS assay quality $\left(Z^{\prime}=0.80 \pm 0.01\right)^{21}$. We incubated test compounds with live cells expressing the SERCA2a-PLB biosensor in a 384-well assay format ${ }^{36}$, and selected Hits that changed FLT (>3S.D.) of the GFP donor fused to SERCA2a in triplicate screens of the library (red points in Fig. 4a). The distribution of FLT for the DMSO-control wells is normal and centers at $2.30 \mathrm{~ns}$ (Fig. 4b). Because FLT measurements are susceptible to interference from fluorescence of the test compound itself, we also acquired fluorescence emission spectra by scanning the same 384 -well plates using a recently developed spectral plate reader ${ }^{32}$ (Fig. 4c) and applied a spectral similarity index to flag fluorescent compounds $s^{36,37}$. In total, 21 compounds were identified as Hits, which altered FRET from SERCA2a to PLB in at least 2 replicates after filtering out compounds with interfering fluorescence (false positives). This equates to a $\sim 1.6 \%$ Hit rate, which is in the acceptable range between $0.5-3 \%$ to maintain a manageable number for post-HTS testing through orthogonal assays ${ }^{38}$.

FRET concentration-response assays. The Hit compounds were further tested in concentration-response (0.01-100 $\mu \mathrm{M}$ compound) FRET assays under the same conditions as used in the primary screens, and $17 \mathrm{com}-$ pounds displayed concentration-dependent changes in FRET (Table S1). Of these, 5 compounds (tenidap, FPL 64176, CP 135807, olprinone, and X80) did not reach saturation of FRET, as evidenced by high $\mathrm{EC}_{50}$ values $(>100 \mu \mathrm{M})$ when fit to Eq. (13). It is possible that these compounds are not intrinsically fluorescent but are affecting the GFP FLT by changing the fluorophore environment or structure. The remaining 12 compounds had $\mathrm{EC}_{50}$ values below $100 \mu \mathrm{M}$ (Fig. 5a, Figure S3, Table S1) and cause either an increase in FRET (e.g., Ro 41-0960) or decrease in FRET (e.g., CP 154526, ivermectin, SCH-202676) of the SERCA2a-PLB biosensor. Thus, the FRET dose-response assay can function as an effective tool to refine the list of Hits and limit the number of compounds to be tested via medium-throughput activity assays in $>50 \mathrm{~K}$ compound screens.

Functional effects of structural (FRET) Hits on Ca-ATPase function. To characterize the relationship between the structural readout (FRET) and the functional effects, in vitro Ca-ATPase assays were performed on cardiac SR (which contains both SERCA2a and PLB). During diastole and systole, the intracellular Ca concentration oscillates between 0.1 and $1 \mu \mathrm{M}$ (pCa 7 to pCa 6) ${ }^{4}$. PLB does not alter basal or maximal SERCA2a activity but inhibits ATPase activity within this range of Ca concentration. An initial functional screen to identify compounds that disrupt PLB inhibition was carried out by measuring Ca-ATPase activity at an intermediate Ca concentration $(\mathrm{pCa}=6.4)$. Ro 41-0960 increased activity by $44 \%($ at $24 \mu \mathrm{M})$ in cardiac SR in this Ca-ATPase assay (Fig. 5b), while 8 compounds were inhibitors under these conditions. 
To further examine the functional effects of Hit compounds, Ca-ATPase assays were performed on both skeletal SR (SERCA1a only) and cardiac SR (SERCA2a + PLB) at a series of Ca concentrations ranging from pCa 5 to 8 . We expect two classes of activators to be useful for the treatment of HF: (1) a compound that increases SERCA's apparent $\mathrm{Ca}$ affinity $\left(\mathrm{pK}_{\mathrm{Ca}}\right.$ ) due to relief of PLB inhibition, which would display a leftward shift in the $\mathrm{V}$ vs. pCa plot in cardiac SR only and (2) a compound that increases $\mathrm{V}_{\max }$ (SERCA activity at saturating Ca). We tested all 21 Hits identified in the HTS-assay for Ca-ATPase activity and found 10 Hits that reproducibly affect SERCA activity (2 activators and 8 inhibitors). The activators included Ro 41-0960 and CP-154526 (chemical structures in Figure S2). Ro 41-0960 increased Ca affinity $\left(\mathrm{pK}_{\mathrm{Ca}}\right)$ in cardiac SR (SERCA2a + PLB) from 6.05 to 6.45 in a concentration-dependent manner $\left(\mathrm{EC}_{50}=22.98 \mu \mathrm{M}\right)$, but had no effect on Ca affinity in skeletal SR (SERCA1a only), even at the highest compound concentrations (Fig. 5c), indicating that the functional effects are specific to the SERCA2a-PLB complex. Notably, SERCA's Ca affinity at saturating [Ro 41-0960] in cardiac SR is equivalent to the Ca affinity in skeletal SR, suggesting that the compound fully reverses PLB inhibition. The second activator discovered, CP-154526, increased $\mathrm{V}_{\max }$ by $34 \%$ in cardiac $\mathrm{SR}\left(\mathrm{EC}_{50}=1.0 \mu \mathrm{M}\right)$ and $35 \%$ in skeletal $\mathrm{SR}\left(\mathrm{EC}_{50}=2.5 \mu \mathrm{M}\right)($ Fig. $5 \mathrm{~d})$.

In addition to the SERCA activators, 8 inhibitor compounds were found in our screen. Based on the design of the assay to detect the SERCA-PLB interaction, we expected that the functional effects of Hit compounds would be to increase the apparent Ca affinity (similar to Ro 41-0960). Surprisingly, the majority (80\%) of Hit compounds that had functional effects were found to inhibit SERCA. The most likely explanation is that these compounds bind to the SERCA2a-PLB complex and induce inhibitory alterations in the structure of the complex, consistent with changes in FRET between fluorophores attached to SERCA2a and PLB. These compounds inhibit SERCA activity in both skeletal (SSR, SERCA1a in the absence of PLB) and cardiac SR (CSR, SERCA2a in the presence of PLB). From the FRET measurements alone, we cannot determine potential binding interfaces of the compound to SERCA, but we speculate that these compounds do not bind to the SERCA2a-PLB interface, but rather affect FRET through allosteric changes in SERCA itself. Also, we cannot rule out the possibility of isoform-dependent activities (SERCA1a vs SERCA2a); although recent crystal structures (PDB ID = 5MPM) demonstrate strong similarity between the isoforms. Figure 6 highlights the results from the two most effective SERCA inhibitors (chemical structures in Figure S2). SCH 202676 decreased $\mathrm{V}_{\max }$ by $68 \%\left(\mathrm{EC}_{50}=1.4 \mu \mathrm{M}\right)$ in cardiac SR and $97 \%$ in skeletal SR $\left(\mathrm{EC}_{50}=0.9 \mu \mathrm{M}\right)$ (Fig. 6a, Figure S4). The antiparasitic ivermectin decreased $\mathrm{V}_{\max }$ by $78 \%\left(\mathrm{EC}_{50}=6.2 \mu \mathrm{M}\right)$ in cardiac SR and $88 \%$ in skeletal SR $\left(\mathrm{EC}_{50}=5.8 \mu \mathrm{M}\right)$ (Figure b, Figure S4). These results agree with a previous report showing inhibition of skeletal muscle SR Ca-ATPase by ivermectin ${ }^{39}$, with $90 \%$ of the activity being inhibited at high concentrations $(50 \mu \mathrm{M})$. Overall, there is strong correlation between structural (FRET-based) and functional (Ca-ATPase-based) results. Moreover, we found a diversity of chemotypes (Figure S2) and functional outcomes of the tested Hit compounds.

\section{Discussion}

The Ca re-uptake process is deficient in cardiomyocytes in both experimental and human heart failure ${ }^{40-44}$. Therapies targeting the underlying molecular causes have the potential to significantly restore heart function ${ }^{6,7}$. SERCA2a sequesters Ca into the SR of cardiomyocytes, and its function is regulated through protein-protein interactions with $\mathrm{PLB}^{3}$. A decrease in expression of SERCA2a is a hallmark of heart failure, and gene therapy approaches to restore its levels back to normal have been successful in experimental models but not so far in clinical trials ${ }^{13,16,17,45-47}$. The relevance of the SERCA2a-PLB complex in human heart function is underscored by the existence of patients who develop cardiomyopathies due to mutations in the PLN (PLB-encoding) gene ${ }^{48-50}$. Some of the PLN mutations have opposite sub-cellular mechanisms of action. One of them, R9C, results in chronic inhibition of SERCA2a by mutant PLB and early death, which is consistent with the known mechanisms on PLB regulation and the findings in mice ${ }^{48,51}$. The other mutation is associated with loss of PLB function (L39stop) and results in dilated cardiomyopathy and premature death in the homozygous, opposite to what is seen in mice ${ }^{50}$. The interaction between SERCA2a and PLB is complex and may be species dependent. Despite great importance, the development of SERCA2a-PLB specific therapies has been limited, largely due to the lack of sensitive and rapid methods for screening compound libraries that disrupt or alter the protein-protein interface.

The present report addresses this problem through the development of a structure-based HTS method using the GFP-RFP FRET pair fused to the human isoforms of SERCA2a and PLB, expressed in live cells. Of the 10 Hits that reproducibly altered SERCA2a-PLB structure and function in this report, most of the compounds (9) have not been previously identified to affect Ca-ATPase activity. The one exception is ivermectin, a broad-spectrum antiparasitic compound. Although it is non-toxic to animals at applied doses, it has been reported that ivermectin, along with other macrocyclic lactones, inhibits SERCA activity at higher doses ${ }^{39}$. Ivermectin appears to inhibit all SERCA isoforms $\left(1 \mathrm{a}^{39}, 2 \mathrm{a}^{52}\right.$, and $\left.2 \mathrm{~b}^{39}\right)$, as well other mammalian P-type ATPases $\left(\mathrm{Na}^{+}, \mathrm{K}^{+}\right.$and $\mathrm{H}^{+}$/ $\mathrm{K}^{+}$). Our results support these findings, as we see inhibition of skeletal SR (containing SERCA1a) and cardiac SR (containing SERCA2a and PLB) with $\mathrm{EC}_{50}$ values of $5.8 \mu \mathrm{M}$ and $6.2 \mu \mathrm{M}$, respectively. Most importantly, two of the Hit compounds identified are SERCA activators. These include Ro 41-0960, which increases the apparent Ca affinity of SERCA2a, as seen in a leftward shift in the Ca-ATPase assay data, and CP 154526, which increases the enzyme's maximal activity at high [Ca]. This confirms that this live-cell HTS platform can detect compounds that act to alter the SERCA2a-PLB interaction and also compounds that act on SERCA independently of PLB.

SERCA1a is also subject to regulation by sarcolipin (SLN) in skeletal muscle. SLN functions by decreasing $\mathrm{Ca}$ affinity of SERCA and partially uncoupling Ca transport from ATP hydrolysis, and is proposed to bind to a similar transmembrane interface of SERCA as PLB (groove formed by M2, M6, and M9 helices) with distinct orientations of the PLB/SLN cytoplasmic domains. Of the compounds tested in this pilot screen, no compound had effects on SSR exclusively that would be expected upon changes in the SERCA1a/SLN interaction, such as increased Ca affinity. Future studies with RFP-labeled SLN may be needed to discover such compounds. 
The advancement of fluorescent-protein biosensors as tools for drug discovery has generated new classes of reagents that report specific molecular processes within the intracellular milieu that have been previously inaccessible to HTS ${ }^{53}$. FRET techniques are sensitive to changes in intra- and inter- molecular distances and can thus report relatively small changes in protein structures and protein-protein interactions. As a result, cell-based FRET assays have been developed to monitor a range of biological activities including membrane and cytoplasmic processes $^{54,55}$. The application of FLT measurements and analysis allows the detection of subtle FRET changes that would be missed by intensity-based technology $y^{20,21,56}$. Indeed, the majority of our Hit compounds caused small FRET efficiency changes, ranging 0.02 to 0.06 . The sensitivity of the assay to compounds of the SERCA2a-PLB functional interaction is indicated by the observation that $\sim 50 \%$ of compounds tested that altered SERCA2a-PLB FRET in a concentration-dependent manner also directly affected SERCA function, as measured by an in vitro ATPase assay.

The position of the fluorophore tagged to a protein can alter its function and may not report the exact position of certain domains of the tagged protein. This concern is especially relevant for FPs because of their large size. On the other hand, the fact that the fluorophore of GFP and RFP is buried within the barrel allows the fluorophore to maintain a homogenous chemical environment. Attachment of FPs to SERCA and PLB did not alter catalytic activity nor its ability to localize to the membrane ${ }^{23}$, including the GFP-RFP FRET pair used in this study ${ }^{30}$. Still, it is necessary to understand that FRET measurements report the proximity between fluorophore tags and only indirectly on the connected protein domain arrangement. Because it is possible that the position of the GFP and/or RFP molecules are affected by assay conditions or the presence of test compounds, addition controls are needed. It is also possible that compounds may be able to quench the donor fluorescence without emitting detectable fluorescence themselves, as would be detected in the visible spectrum (Fig. 4c). As a reference for this possibility, we have screened the LOPAC library under similar conditions (e.g., final [compound] $=10 \mu \mathrm{M}) \mathrm{using}$ HEK cells expressing the GFP-RFP FRET pair connected by a flexible linker or GFP-SERCA2a (donor) alone. Compounds that altered the FLT greater than 3 S.D. in these controls were excluded from further consideration, and not included in the 21 Hit compounds reported here. Lastly, separation distance changes or modulation in dipole-dipole interactions can engender changes in FRET. Because the rotational correlation time of FPs is longer than observed fluorescent decays, this brings into question whether to assume dynamically averaged dipole-dipole orientations (e.g., $\kappa^{2}=2 / 3$ ) or a static isotropic model ${ }^{57}$. Vogel et al., compares the dependence of $<\mathrm{E}>$ for CFP on the assumption of dynamic and static models, and demonstrates that the apparent FRET values observed (between $0.10-0.15$ ) for the SERCA2a-PLB biosensor are similar assuming either model ${ }^{58}$. Further, the $\kappa^{2}$ parameter was calculated by simulation for a SERCA molecule with CFP attached at the N-terminus using a similar length linker as used in this study ${ }^{59}$. The orientation factor is 0.6 , which is slightly lower than what is predicted by a dynamic model ${ }^{59}$.

Considering this, the Hit compounds identified cause relatively small changes in the FRET readout and do not eliminate the FRET signal, implying that subtle domain movement of SERCA2a and/or PLB is sufficient to influence enzymatic function. This also suggests that these compound-dependent conformational changes occur while PLB is still bound to SERCA2a. This is consistent with reports detecting at least two distinct PLB conformations exist while bound to SERCA, each with opposing functional outcomes (Subunit Model) ${ }^{27,31,60-62}$.

All these compounds are different from those identified in an earlier screen, where we used a 2-color SERCA construct $^{21,36,63}$. Thus, this SERCA2a-PLB HTS assay could be used in combination with the SERCA-only assays, to enrich the spectrum of SERCA-modulator chemotypes. More generally, the approach illustrated here is applicable to any protein-protein interaction target for which there is sufficient information about structure and function to design FRET biosensors that are sensitive to changes in the protein complex interactions.

\section{Methods}

Molecular Biology. eGFP and tagRFP were fused to the N-terminus of human SERCA2a and human PLB, respectively, as described previously ${ }^{21,36,64}$. We have previously demonstrated that attachment of the fluorescent proteins at these sites does not interfere with the activity of SERCA ${ }^{21,63}$. Native PLB equilibrates between monomers and homopentamers ${ }^{65}$. To simplify the system and ensure that we are specifically measuring the SERCA2aPLB interaction, a monomeric mutant of $\mathrm{PLB}^{24}$ was used, with three mutations (C36A, C41A, and C46A) in the transmembrane domain. All mutations were introduced by Quikchange mutagenesis (Agilent Technologies, Santa Clara, CA) and sequenced for confirmation.

Cell Culture. Human embryonic kidney cells 293 (HEK293, ATCC, Manassas, VA) were cultured in phenol red-free Dulbecco's modified Eagle's medium (DMEM) (Gibco, Waltham, MA) supplemented with 2 mM l-glutamine (Invitrogen, Waltham, MA), heat-inactivated 10\% fetal bovine serum (FBS HI, Gibco). Cell cultures were maintained in an incubator with 5\% CO2 (Forma Series II Water Jacket CO2 Incubator, Thermo Fisher Scientific, Waltham, MA) at $37^{\circ} \mathrm{C}$. To generate the one color human SERCA2a stable cell line, HEK293 cells were transiently transfected using Lipofectamine 3000 (Invitrogen). Transiently transfected cells were treated with G418 (Sigma-Aldrich, St. Louis, MO) for two weeks to select for expressing cells. Stable cell lines expressing 2C-SERCA were screened for uniform population by flow cytometry and fluorescence microscopy. To generate SERCA2a-PLB biosensor, HEK293 cells were transiently transfected using Lipofectamine 3000 with GFP-SERCA2a and RFP-PLB in a 1:7 molar ratio for the screen. Cells were then assayed 48 hours post-transfection. For cell permeabilization experiments, cells were trypsinized (TrypLE, Thermo Fischer Scientific), PBS-washed (three times), and resuspended in a homogenization buffer (20 mM MOPS, $0.5 \mathrm{MgCl}_{2}$, protease inhibitor cocktail (Roche, Basel, Switzerland)). Saponin (Sigma-Aldrich) was added to a final concentration of $10 \mu \mathrm{g} / \mathrm{mL}$ and cells were incubated for $4 \mathrm{~min}$ at $37^{\circ} \mathrm{C}$ water bath. Saponin was removed by centrifugation for $3 \mathrm{~min}$, cells were washed twice with homogenization buffer, and resuspended in homogenization buffer. 
Western Blot. Samples were separated on a 4-20\% polyacrylamide gradient gel (Bio-Rad, Hercules, CA) and transferred to polyvinylidene difluoride membrane. The membrane was blocked in Odyssey Blocking Buffer (LI-COR Biosciences, Lincoln, NE) followed by overnight incubation of the primary antibody rabbit anti-GFP (1:1000; ab290, Abcam, Cambridge, United Kingdom $)^{66}$, mouse anti-SERCA2 (1:1000; 2A7-A1, Abcam $)^{21}$, rabbit anti-tagRFP (1:1000; ab233, Evrogen $)^{67}$, mouse anti-PLB (1:1000, 2D12, Abcam $)^{64}$ or rabbit anti- $\beta$-actin $(1: 5000$, ab8227, Abcam $)^{68}$ in the cold room. Blots were incubated with anti-mouse or anti-rabbit secondary antibodies conjugated to IRDye $680 \mathrm{RD}$ or IRDye $800 \mathrm{CW}$, respectively, for $1 \mathrm{~h}$ at room temperature $(1: 20,000$; LI-COR Biosciences). Blots were quantified on the Odyssey scanner (LI-COR Biosciences). Full-length blots are presented in Supplementary Figure S5.

Compound Handling and Preparation of 384-Well Assay Plates. The LOPAC compounds (Thermo Fisher Scientific) were received in 96-well plates and reformatted into 384-well polypropylene intermediate plates (Greiner Bio-One, Kremsmunster, Austria) using a multichannel liquid handler, BioMek FX (Beckman Coulter, Miami, FL), and then transferred to 384-well Echo Qualified source plates (Labcyte, Inc., Sunnyvale, CA). Assay plates were prepared by transferring $50 \mathrm{~nL}$ of the $10 \mathrm{mM}$ compound stocks or DMSO from the source plates to 384-well black polypropylene plates (Greiner), using an Echo 550 acoustic dispenser (Labcyte). LOPAC compounds were formatted in four plates, with the first two and last two columns loaded with $50 \mathrm{~nL}$ of DMSO and used for compound-free controls. These assay plates were then sealed and stored at $-20^{\circ} \mathrm{C}$ prior to usage. Cells were dispensed $(50 \mu \mathrm{L})$ using a Multidrop Combi liquid dispenser from Thermo (Pittsburg, PA), at a density of $10^{6}$ cells $/ \mathrm{mL}$ in PBS.

Fluorescence Data Acquisition. FLT measurements were conducted in a prototype top-read FLT-PR designed and built by Fluorescence Innovations, which reads each 384-well plate in $\sim 3 \mathrm{~min}$. GFP donor fluorescence was excited with a $473 \mathrm{~nm}$ microchip laser from Concepts Research Corporation (Belgium, WI), and emission was acquired with $490 \mathrm{~nm}$ long-pass and 520/17 nm band-pass filters (Semrock, Rochester, NY). This instrument uses a unique direct waveform recording technology that enables high-throughput FLT detection at high precision ${ }^{69}$. We have previously demonstrated the performance of this plate reader with known fluorescence standards, as well as with a FRET-based HTS method that targets SERCA ${ }^{22,36,37}$.

HTS Data Analysis. TR-fluorescence waveforms for each well were fitted based on a two-exponential decay function using least-squares minimization global analysis software ${ }^{22}$. FRET efficiency (E) was determined as the fractional decrease of donor FLT $\left(\tau_{D}\right)$, due to the presence of acceptor fluorophore $\left(\tau_{D A}\right)$ :

$$
E=1-\frac{\tau_{D A}}{\tau_{D}}
$$

Assay quality was determined based on controls (DMSO-only samples) on each plate, as indexed by the $\mathrm{Z}^{\prime}$ factor $^{35}$

$$
Z^{\prime}=1-\frac{3\left(\sigma_{D A}+\sigma_{D}\right)}{\left|\mu_{D A}-\mu_{D}\right|}
$$

where $\sigma_{\mathrm{D}}$ and $\sigma_{\mathrm{DA}}$ are the standard deviations (SDs) of the controls $\tau_{\mathrm{D}}$ and $\tau_{\mathrm{DA}}$, respectively, and $\mu_{\mathrm{D}}$ and $\mu_{\mathrm{DA}}$ are the means of the controls $\tau_{D}$ and $\tau_{D A}$, respectively. A compound was considered a Hit if it changed E by $>3 S D$ relative to that of control samples $\left(\mathrm{E}_{0}\right)$ that were exposed to $0.1 \%$ DMSO. The 3 SD Hit selection threshold is typical for normally distributed HTS data, whereby $0.27 \%$ of the readings are expected to fall outside this limit. The threshold may be further adjusted to constrain the number of Hits according to the resources available for evaluation via secondary (orthogonal) assays.

Time-Resolved FRET. TRF waveforms from donor and FRET-labeled samples were analyzed as described in our previous publications ${ }^{69-71}$. The measured time-resolved fluorescence waveform,

$$
I(\boldsymbol{t})=\int_{-\infty}^{\infty} \operatorname{IRF}\left(t-t^{\prime}\right) \cdot F\left(t^{\prime}\right) d t^{\prime}
$$

is a function of the nanosecond decay time $t$, and is modeled as the convolution integral of the measured instrument response function, $\operatorname{IRF}(\mathrm{t})$, and the fluorescence decay model, $F(\mathrm{t})$. The fluorescence decay model

$$
F_{D+A}(t)=x_{D} F_{D}(t)+\left(1-x_{D}\right) F_{D A}(t)
$$

is a linear combination of a donor-only fluorescence decay function $F_{\mathrm{D}}(\mathrm{t})$ and an energy transfer-affected donor fluorescence decay $F_{\mathrm{DA}}(\mathrm{t})$. The donor decay $F_{\mathrm{D}}(\mathrm{t})$ is a sum of $n$ exponentials

$$
F_{D}(t)=\sum_{i=1}^{n} A_{i} \exp \left(-t / \tau_{i}\right)
$$

with discrete FLT species $\tau_{\mathrm{i}}$ and pre-exponential mole fractions $A_{\mathrm{i}}$. For the GFP donor, two exponentials $(n=2)$ were required to fit the observed fluorescence. The energy transfer-affected donor decay function, $F_{\mathrm{DA}}(\mathrm{t})$, 


$$
F_{D A}(t)=\sum_{j=1}^{N} X_{j} \cdot T_{j}(t)
$$

is a sum over multiple structural states $(j)$ with mole fractions $X_{\mathrm{j}}$, represented by FRET-affected donor fluorescence decays $T_{\mathrm{j}}(\mathrm{t})$. The increase in the donor decay rate (inverse donor FLT) due to FRET is given by the Förster equation

$$
k_{T i}=k_{D i}\left(R / R_{0 i}\right)^{-6} \text {, }
$$

where

$$
k_{\mathrm{DAi}}=k_{\mathrm{Di}}+k_{\mathrm{Ti}}
$$

and

$$
k_{D i}=1 / \tau_{i}
$$

We modeled TR-FRET assuming that each structural state $j$ (Eq. (6) corresponds to a Gaussian distribution of interprobe distances, $\rho_{\mathrm{j}}(R)$ :

$$
\begin{gathered}
T_{j}(t)=\int_{-\infty}^{\infty} \rho_{j}(R) \cdot \sum_{i=1}^{n} A_{i} \exp \left(\frac{-t}{\tau_{i}} \cdot\left[1+\left(\frac{R_{0 i}}{R}\right)^{6}\right]\right) d R \\
\rho_{\mathrm{j}}(R)=\frac{1}{\sigma_{\mathrm{j}} \sqrt{2 \pi}} \exp \left(\frac{-\left[R-R_{\mathrm{j}}\right]^{2}}{2 \sigma_{\mathrm{j}}^{2}}\right) \\
\sigma_{j}=F_{W H M_{j} /(2 \sqrt{2 \ln 2})}
\end{gathered}
$$

Isolation of Sarcoplasmic Reticulum Vesicles. Skeletal muscle SR membrane vesicles were isolated from longissimus dorsi obtained from New Zealand white rabbits, as previously described ${ }^{31}$. Cardiac SR membrane vesicles were isolated from ventricular myocardium obtained from fresh pig hearts ${ }^{72}$. All experimental protocols were reviewed and approved by University of Minnesota's Institutional Animal Care and Use Committee, an Association for Assessment and Accreditation of Laboratory Animal Care institution.

Ca-ATPase Activity. An enzyme-coupled, NADH-linked ATPase assay was used to measure SERCA ATPase activity in 96-well microplates. Each well contained $0.8 \mu \mathrm{g}$ (skeletal) or $2 \mu \mathrm{g}$ (cardiac) of SR vesicles adjusted for the different SERCA contents of skeletal and cardiac SR, $50 \mathrm{mM}$ MOPS (pH 7.0), $100 \mathrm{mM} \mathrm{KCl,} 5 \mathrm{mM} \mathrm{MgCl}_{2}$, $1 \mathrm{mM}$ EGTA, $0.2 \mathrm{mM}$ NADH, $1 \mathrm{mM}$ phosphoenol pyruvate, $10 \mathrm{IU} / \mathrm{mL}$ of pyruvate kinase, $10 \mathrm{IU} / \mathrm{mL}$ of lactate dehydrogenase, $3.5 \mu \mathrm{g} / \mathrm{mL}$ of the $\mathrm{Ca}$ ionophore $\mathrm{A} 23187$, and $\mathrm{CaCl}_{2}$ added to set free [Ca] to the desired values. The assay was started upon the addition of ATP at a final concentration of $5 \mathrm{mM}$ and read in a SpectraMax Plus microplate spectrophotometer rom Molecular Devices (Sunnyvale, CA). The Ca-ATPase assays were conducted over a range of $[\mathrm{Ca}]$, and the ATPase activities were fitted using the Hill function

$$
V=\frac{V_{\max }}{1+10-\left(n\left(p K_{c a}-p C a\right)\right)}
$$

where $\mathrm{V}$ is the initial ATPase rate, $\mathrm{V}_{\text {max }}$ is the ATPase at saturating [Ca], $\mathrm{n}$ is the Hill coefficient, and $\mathrm{pK} \mathrm{Ca}_{\mathrm{a}}$ is the apparent $\mathrm{Ca}$ dissociation constant.

Statistical Analysis. Errors are reported as the standard deviation of the mean, except when noted, and statistical significance was determined by Student's T test, where $\mathrm{p}<0.05$ was considered significant, or 1-way ANOVA followed by Tukey post hoc analysis.

Data availability. The datasets generated during and/or analyzed during the current study are available from the corresponding author on reasonable request.

\section{References}

1. Arkin, M. R. \& Wells, J. A. Small-molecule inhibitors of protein-protein interactions: progressing towards the dream. Nat Rev Drug Discov 3, 301-317, https://doi.org/10.1038/nrd1343 (2004).

2. Wells, J. A. \& McClendon, C. L. Reaching for high-hanging fruit in drug discovery at protein-protein interfaces. Nature 450, 1001-1009, https://doi.org/10.1038/nature06526 (2007).

3. Eisner, D. A., Caldwell, J. L., Kistamas, K. \& Trafford, A. W. Calcium and Excitation-Contraction Coupling in the Heart. Circulation research 121, 181-195, https://doi.org/10.1161/CIRCRESAHA.117.310230 (2017)

4. MacLennan, D. H. \& Kranias, E. G. Phospholamban: a crucial regulator of cardiac contractility. Nat Rev Mol Cell Biol 4, 566-577, https://doi.org/10.1038/nrm1151 (2003) 
5. Bers, D. M. Cardiac excitation-contraction coupling. Nature 415, 198-205, https://doi.org/10.1038/415198a (2002).

6. Lipskaia, L., Chemaly, E. R., Hadri, L., Lompre, A. M. \& Hajjar, R. J. Sarcoplasmic reticulum $\mathrm{Ca}(2+)$ ATPase as a therapeutic target for heart failure. Expert Opin Biol Ther 10, 29-41, https://doi.org/10.1517/14712590903321462 (2010).

7. Lompre, A. M. et al. Ca2+ cycling and new therapeutic approaches for heart failure. Circulation 121, 822-830, https://doi. org/10.1161/CIRCULATIONAHA.109.890954 (2010).

8. Schwinger, R. H. et al. Unchanged protein levels of SERCA II and phospholamban but reduced Ca2+ uptake and $\mathrm{Ca}(2+)$-ATPase activity of cardiac sarcoplasmic reticulum from dilated cardiomyopathy patients compared with patients with nonfailing hearts. Circulation 92, 3220-3228 (1995).

9. Gwathmey, J. K. et al. Abnormal intracellular calcium handling in myocardium from patients with end-stage heart failure. Circulation research 61, 70-76 (1987).

10. Hasenfuss, G. Alterations of calcium-regulatory proteins in heart failure. Cardiovasc Res 37, 279-289 (1998).

11. Hasenfuss, G. Animal models of human cardiovascular disease, heart failure and hypertrophy. Cardiovasc Res 39, 60-76 (1998).

12. Hoshijima, M. et al. Chronic suppression of heart-failure progression by a pseudophosphorylated mutant of phospholamban via in vivo cardiac rAAV gene delivery. Nat Med 8, 864-871, https://doi.org/10.1038/nm739 (2002).

13. Miyamoto, M. I. et al. Adenoviral gene transfer of SERCA2a improves left-ventricular function in aortic-banded rats in transition to heart failure. Proceedings of the National Academy of Sciences of the United States of America 97, 793-798 (2000).

14. Schmidt, U. et al. Restoration of diastolic function in senescent rat hearts through adenoviral gene transfer of sarcoplasmic reticulum $\mathrm{Ca}(2+)$-ATPase. Circulation 101, 790-796 (2000).

15. Byrne, M. J. et al. Recirculating cardiac delivery of AAV2/1SERCA2a improves myocardial function in an experimental model of heart failure in large animals. Gene Ther 15, 1550-1557, https://doi.org/10.1038/gt.2008.120 (2008).

16. Jessup, M. et al. Calcium Upregulation by Percutaneous Administration of Gene Therapy in Cardiac Disease (CUPID): a phase 2 trial of intracoronary gene therapy of sarcoplasmic reticulum Ca2+-ATPase in patients with advanced heart failure. Circulation 124, 304-313, https://doi.org/10.1161/CIRCULATIONAHA.111.022889 (2011).

17. Zsebo, K. et al. Long-term effects of AAV1/SERCA2a gene transfer in patients with severe heart failure: analysis of recurrent cardiovascular events and mortality. Circulation research 114, 101-108, https://doi.org/10.1161/CIRCRESAHA.113.302421 (2014).

18. Greenberg, B. et al. Prevalence of AAV1 neutralizing antibodies and consequences for a clinical trial of gene transfer for advanced heart failure. Gene Ther 23, 313-319, https://doi.org/10.1038/gt.2015.109 (2016).

19. Johnson, R. G. Jr. Pharmacology of the cardiac sarcoplasmic reticulum calcium ATPase-phospholamban interaction. Annals of the New York Academy of Sciences 853, 380-392 (1998).

20. Cornea, R. L. et al. High-Throughput FRET Assay Yields Allosteric SERCA Activators. J Biomol Screen, 1087057112456878 https:// doi.org/10.1177/1087057112456878 (2012).

21. Gruber, S. J. et al. Discovery of enzyme modulators via high-throughput time-resolved FRET in living cells. J Biomol Screen 19, 215-222, https://doi.org/10.1177/1087057113510740 (2014).

22. Petersen, K. J. et al. Fluorescence lifetime plate reader: resolution and precision meet high-throughput. Rev Sci Instrum 85, 113101, https://doi.org/10.1063/1.4900727 (2014).

23. Bidwell, P., Blackwell, D. J., Hou, Z., Zima, A. V. \& Robia, S. L. Phospholamban binds with differential affinity to calcium pump conformers. J Biol Chem 286, 35044-35050, https://doi.org/10.1074/jbc.M111.266759 (2011).

24. Karim, C. B., Kirby, T. L., Zhang, Z., Nesmelov, Y. \& Thomas, D. D. Phospholamban structural dynamics in lipid bilayers probed by a spin label rigidly coupled to the peptide backbone. Proc Natl Acad Sci USA 101, 14437-14442 (2004).

25. Zamoon, J., Mascioni, A., Thomas, D. D. \& Veglia, G. NMR solution structure and topological orientation of monomeric phospholamban in dodecylphosphocholine micelles. Biophys J 85, 2589-2598, https://doi.org/10.1016/S0006-3495(03)74681-5 (2003).

26. Lockwood, N. A. et al. Structure and function of integral membrane protein domains resolved by peptide-amphiphiles: application to phospholamban. Biopolymers 69, 283-292, https://doi.org/10.1002/bip.10365 (2003).

27. Zamoon, J., Nitu, F., Karim, C., Thomas, D. D. \& Veglia, G. Mapping the interaction surface of a membrane protein: Unveiling the conformational switch of phospholamban in calcium pump regulation. Proc Natl Acad Sci USA 102, 4747-4752 (2005).

28. Karim, C. B., Zhang, Z., Howard, E. C., Torgersen, K. D. \& Thomas, D. D. Phosphorylation-dependent Conformational Switch in Spin-labeled Phospholamban Bound to SERCA. J Mol Biol 358, 1032-1040 (2006).

29. Li, J., James, Z. M., Dong, X., Karim, C. B. \& Thomas, D. D. Structural and functional dynamics of an integral membrane protein complex modulated by lipid headgroup charge. J Mol Biol 418, 379-389, S0022-2836(12)00152-0 https://doi.org/10.1016/j. jmb.2012.02.011 (2012)

30. Blackwell, D. J., Zak, T. J. \& Robia, S. L. Cardiac Calcium ATPase Dimerization Measured by Cross-Linking and Fluorescence Energy Transfer. Biophys J 111, 1192-1202, https://doi.org/10.1016/j.bpj.2016.08.005 (2016)

31. Mueller, B., Karim, C. B., Negrashov, I. V., Kutchai, H. \& Thomas, D. D. Direct detection of phospholamban and sarcoplasmic reticulum Ca-ATPase interaction in membranes using fluorescence resonance energy transfer. Biochemistry 43, 8754-8765 (2004).

32. Li, J., Xiong, Y., Bigelow, D. J. \& Squier, T. C. Phospholamban binds in a compact and ordered conformation to the Ca-ATPase. Biochemistry 43, 455-463, https://doi.org/10.1021/bi035424v (2004).

33. Augustin, J. M., Kuzina, V., Andersen, S. B. \& Bak, S. Molecular activities, biosynthesis and evolution of triterpenoid saponins. Phytochemistry 72, 435-457, https://doi.org/10.1016/j.phytochem.2011.01.015 (2011).

34. Dandapani, S., Rosse, G., Southall, N., Salvino, J. M. \& Thomas, C. J. Selecting, Acquiring, and Using Small Molecule Libraries for High-Throughput Screening. Curr Protoc Chem Biol 4, 177-191, https://doi.org/10.1002/9780470559277.ch110252 (2012).

35. Zhang, J. H., Chung, T. D. \& Oldenburg, K. R. A Simple Statistical Parameter for Use in Evaluation and Validation of High Throughput Screening Assays. J Biomol Screen 4, 67-73, https://doi.org/10.1177/108705719900400206 (1999).

36. Schaaf, T. M. et al. High-Throughput Spectral and Lifetime-Based FRET Screening in Living Cells to Identify Small-Molecule Effectors of SERCA. SLAS Discov 22, 262-273, https://doi.org/10.1177/1087057116680151 (2017).

37. Schaaf, T. M., Peterson, K. C., Grant, B. D., Thomas, D. D. \& Gillispie, G. D. Spectral Unmixing Plate Reader: High-Throughput, High-Precision FRET Assays in Living Cells. SLAS Discov 22, 250-261, https://doi.org/10.1177/1087057116679637 (2017).

38. Hughes, J. P., Rees, S., Kalindjian, S. B. \& Philpott, K. L. Principles of early drug discovery. Br J Pharmacol 162, 1239-1249, https:// doi.org/10.1111/j.1476-5381.2010.01127.x (2011).

39. Bilmen, J. G., Wootton, L. L. \& Michelangeli, F. The inhibition of the sarcoplasmic/endoplasmic reticulum Ca2+-ATPase by macrocyclic lactones and cyclosporin A. Biochem J 366, 255-263, https://doi.org/10.1042/BJ20020431 (2002).

40. Morgan, J. P., Erny, R. E., Allen, P. D., Grossman, W. \& Gwathmey, J. K. Abnormal intracellular calcium handling, a major cause of systolic and diastolic dysfunction in ventricular myocardium from patients with heart failure. Circulation 81, III21-32 (1990).

41. Schmidt, U. et al. Contribution of abnormal sarcoplasmic reticulum ATPase activity to systolic and diastolic dysfunction in human heart failure. J Mol Cell Cardiol 30, 1929-1937, https://doi.org/10.1006/jmcc.1998.0748 (1998).

42. Hasenfuss, G. et al. Calcium handling proteins in the failing human heart. Basic Res Cardiol 92(Suppl 1), 87-93 (1997).

43. Hasenfuss, G. et al. Relation between myocardial function and expression of sarcoplasmic reticulum $\mathrm{Ca}(2+)$-ATPase in failing and nonfailing human myocardium. Circ Res 75, 434-442 (1994).

44. Hasenfuss, G. et al. Calcium cycling proteins and force-frequency relationship in heart failure. Basic Res Cardiol 91(Suppl 2), 17-22 (1996).

45. del Monte, F. et al. Abrogation of ventricular arrhythmias in a model of ischemia and reperfusion by targeting myocardial calcium cycling. Proc Natl Acad Sci USA 101, 5622-5627, https://doi.org/10.1073/pnas.0305778101 (2004). 
46. del Monte, F. et al. Improvement in survival and cardiac metabolism after gene transfer of sarcoplasmic reticulum $\mathrm{Ca}(2+)$-ATPase in a rat model of heart failure. Circulation 104, 1424-1429 (2001).

47. Kawase, Y. et al. Reversal of cardiac dysfunction after long-term expression of SERCA2a by gene transfer in a pre-clinical model of heart failure. J Am Coll Cardiol 51, 1112-1119, https://doi.org/10.1016/j.jacc.2007.12.014 (2008).

48. Schmitt, J. P. et al. Dilated cardiomyopathy and heart failure caused by a mutation in phospholamban. Science 299, 1410-1413, https://doi.org/10.1126/science.1081578 (2003).

49. Liu, G. S. et al. A novel human R25C-phospholamban mutation is associated with super-inhibition of calcium cycling and ventricular arrhythmia. Cardiovasc Res 107, 164-174, https://doi.org/10.1093/cvr/cvv127 (2015).

50. Haghighi, K. et al. Human phospholamban null results in lethal dilated cardiomyopathy revealing a critical difference between mouse and human. J Clin Invest 111, 869-876, https://doi.org/10.1172/JCI17892 (2003).

51. Nelson, S. E. D. et al. Effects of the Arg9Cys and Arg25Cys mutations on phospholamban's conformational equilibrium in membrane bilayers. Biochim Biophys Acta 1860, 1335-1341, https://doi.org/10.1016/j.bbamem.2018.02.030 (2018).

52. Pimenta, P. H., Silva, C. L. \& Noel, F. Ivermectin is a nonselective inhibitor of mammalian P-type ATPases. Naunyn Schmiedebergs Arch Pharmacol 381, 147-152, https://doi.org/10.1007/s00210-009-0483-z (2010).

53. Giuliano, K. A. \& Taylor, D. L. Fluorescent-protein biosensors: new tools for drug discovery. Trends Biotechnol 16, 135-140, https:// doi.org/10.1016/S0167-7799(97)01166-9 (1998).

54. Rohde, J. A., Thomas, D. D. \& Muretta, J. M. Heart failure drug changes the mechanoenzymology of the cardiac myosin powerstroke. Proc Natl Acad Sci USA 114, E1796-E1804, https://doi.org/10.1073/pnas.1611698114 (2017).

55. Lo, C. H. et al. An Innovative High-Throughput Screening Approach for Discovery of Small Molecules That Inhibit TNF Receptors. SLAS Discov 22, 950-961, https://doi.org/10.1177/2472555217706478 (2017).

56. Rebbeck, R. T. et al. High-Throughput Screens to Discover Small-Molecule Modulators of Ryanodine Receptor Calcium Release Channels. SLAS discovery: advancing life sciences $R$ \& D 22, 176-186, https://doi.org/10.1177/1087057116674312 (2017).

57. Vogel, S. S., Thaler, C. \& Koushik, S. V. Fanciful FRET. Sci STKE 2006, re2, https://doi.org/10.1126/stke.3312006re2 (2006).

58. Vogel, S. S., Nguyen, T. A., van der Meer, B. W. \& Blank, P. S. The impact of heterogeneity and dark acceptor states on FRET: implications for using fluorescent protein donors and acceptors. PLoS One 7, e49593, https://doi.org/10.1371/journal.pone.0049593 (2012).

59. Svensson, B., Autry, J. M. \& Thomas, D. D. Molecular Modeling of Fluorescent SERCA Biosensors. Methods Mol Biol 1377, 503-522, https://doi.org/10.1007/978-1-4939-3179-8_42 (2016).

60. Dong, X. \& Thomas, D. D. Time-resolved FRET reveals the structural mechanism of SERCA-PLB regulation. Biochem Biophys Res Commun 449, 196-201, https://doi.org/10.1016/j.bbrc.2014.04.166 (2014).

61. Pallikkuth, S. et al. Phosphorylated phospholamban stabilizes a compact conformation of the cardiac calcium-ATPase. Biophys $J$ 105, 1812-1821, https://doi.org/10.1016/j.bpj.2013.08.045 (2013).

62. James, Z. M., McCaffrey, J. E., Torgersen, K. D., Karim, C. B. \& Thomas, D. D. Protein-protein interactions in calcium transport regulation probed by saturation transfer electron paramagnetic resonance. Biophys J 103, 1370-1378, https://doi.org/10.1016/j. bpj.2012.08.032 (2012)

63. Hou, Z. et al. Two-color calcium pump reveals closure of the cytoplasmic headpiece with calcium binding. PLoS ONE, accepted (2012).

64. Gruber, S. J., Haydon, S. \& Thomas, D. D. Phospholamban mutants compete with wild type for SERCA binding in living cells. Biochem Biophys Res Commun 420, 236-240, S0006-291X(12)00384-1 https://doi.org/10.1016/j.bbrc.2012.02.125 (2012).

65. Robia, S. L. et al. Forster transfer recovery reveals that phospholamban exchanges slowly from pentamers but rapidly from the SERCA regulatory complex. Circ Res 101, 1123-1129 (2007).

66. Pankiv, S. et al. p62/SQSTM1 binds directly to Atg8/LC3 to facilitate degradation of ubiquitinated protein aggregates by autophagy. J Biol Chem 282, 24131-24145, https://doi.org/10.1074/jbc.M702824200 (2007).

67. Nakamura, N. et al. Endosomes are specialized platforms for bacterial sensing and NOD2 signalling. Nature 509, 240-244, https:// doi.org/10.1038/nature13133 (2014).

68. Aleksunes, L. M., Augustine, L. M., Scheffer, G. L., Cherrington, N. J. \& Manautou, J. E. Renal xenobiotic transporters are differentially expressed in mice following cisplatin treatment. Toxicology 250, 82-88, https://doi.org/10.1016/j.tox.2008.06.009 (2008).

69. Muretta, J. M. et al. High -performance time-resolved fluorescence by direct waveform recording. Rev Sci Instrum 81, 103101103101 - 103101-103108 (2010).

70. Nesmelov, Y. E. et al. Structural kinetics of myosin by transient time-resolved FRET. Proceedings of the National Academy of Sciences of the United States of America 108, 1891-1896, https://doi.org/10.1073/pnas.1012320108 (2011).

71. Muretta, J. M., Petersen, K. J. \& Thomas, D. D. Direct real-time detection of the actin-activated power stroke within the myosin catalytic domain. Proceedings of the National Academy of Sciences of the United States of America 110, 7211-7216, https://doi. org/10.1073/pnas.1222257110 (2013).

72. Fruen, B. R., Bardy, J. M., Byrem, T. M., Strasburg, G. M. \& Louis, C. F. Differential Ca(2+) sensitivity of skeletal and cardiac muscle ryanodine receptors in the presence of calmodulin. American journal of physiology. Cell physiology 279, C724-733 (2000).

\section{Acknowledgements}

Joseph M. Autry provided helpful discussion, and Octavian Cornea, Destiny Ziebol and Sarah Blakely Anderson provided administrative support. Fluorescence measurements were performed using facilities provided by the Biophysical Technology Center, University of Minnesota, and by Fluorescence Innovations, Inc. (Minneapolis, $\mathrm{MN}$ ), where assistance was provided by Benjamin D. Grant and Kurt C. Peterson. DRS was supported by NIH Training Grant T32 AR07612. TMS was supported by NIH Institutional Research and Academic Career Development Award K12 GM119955. This work was supported by grants from NIH to DDT (R01 GM27906, R01 HL129814, and R37 AG26160), RJH (R01 HL129814), and to RLC (R01 HL092097 and R01 HL138539).

\section{Author Contributions}

D.R.S., D.D.T., R.L.C., J.L. and T.M.S. conceived, directed and analyzed all experimental research. D.R.S., D.D.T., R.L.C., R.J.H. and D.K.C. prepared the manuscript. D.R.S., S.L.Y. and K.A.J. performed FRET spectroscopy experiments and activity assays. All authors reviewed the manuscript.

\section{Additional Information}

Supplementary information accompanies this paper at https://doi.org/10.1038/s41598-018-29685-z.

Competing Interests: The authors declared the following potential conflicts of interest with respect to the research, authorship, and/or publication of this article: DDT and RLC hold equity in, and serve as executive officers for, Photonic Pharma LLC. These relationships have been reviewed and managed by the University of Minnesota. Photonic Pharma had no role in this study. 
Publisher's note: Springer Nature remains neutral with regard to jurisdictional claims in published maps and institutional affiliations.

(c) (i) Open Access This article is licensed under a Creative Commons Attribution 4.0 International License, which permits use, sharing, adaptation, distribution and reproduction in any medium or format, as long as you give appropriate credit to the original author(s) and the source, provide a link to the Creative Commons license, and indicate if changes were made. The images or other third party material in this article are included in the article's Creative Commons license, unless indicated otherwise in a credit line to the material. If material is not included in the article's Creative Commons license and your intended use is not permitted by statutory regulation or exceeds the permitted use, you will need to obtain permission directly from the copyright holder. To view a copy of this license, visit http://creativecommons.org/licenses/by/4.0/.

(c) The Author(s) 2018 